\title{
Junge Forschung in Metallurgie und Werkstofftechnik
}

\author{
Johannes Schenk und Bruno Hribernik
}

Leoben, Österreich

Online publiziert 21. Juni 2016

Die Vortragsreihe „Junge Forschung“ war heuer zum fünten Mal in Folge Programmpunkt des jährlichen Forums für Metallurgie- und Werkstofftechnik in Leoben. Junge Forscherinnen und Forscher von allen technischen Universitäten in Österreich - der Montanuniversität Leoben sowie den beiden Technischen Universitäten Wien und Graz - präsentierten am Nachmittag des 10. Mai 2016 ihre Dissertationen. In diesem Sonderheft sind fünf der präsentierten Vorträge als Originalbeiträge für Sie, liebe Leserinnen und Leser, zum Nachlesen publiziert.

Der erste Beitrag ist aus der metallurgischen Prozesstechnik und beschäftigt sich mit der mathematischen Modellierung der induktiven Erwärmung von Graphitschüttungen für den RecoPhos Prozess, einem Prozess zur Rückgewinnung von Phosphor aus Klärschlammaschen. Die vier folgenden Beiträge in diesem Heft präsentieren Ergebnisse von Arbeiten auf dem Gebiet der Werkstofftechnik. Die Themen umfassen ein weites Spektrum von Problemstellungen, wie systematische Untersuchungen zur Bildung von Azikularferrit in Stählen, die mikrostrukturelle Charakterisierung von ultra-hochfesten Schweißgütern, eine experimentelle und computergestützte Studie zur Initiierung definierter Mikrostrukturen in lichtbogenverdampften $(\mathrm{Al}, \mathrm{Cr})_{2} \mathrm{O}_{3}$ Schutzschichten sowie Untersuchungen mit insitu Methoden zur Ausscheidungs- und Entfestigungskinetik an schnellerstarrten Al-Mg-Sc-Zr Legierungen.
Erfreulich ist das hohe wissenschaftliche und technische Niveau, mit dem sich Dissertationen beschäftigen. Am Forum wurde dies vom anwesenden Publikum bereits äußerst positiv aufgenommen. Wir hoffen, mit diesem Sonderheft einem weiteren Kreis von Fachleuten die Leistungen unseres wissenschaftlichen Nachwuchses näher bringen zu können.

Mit freundlichen Glückauf,

Johannes Schenk

Leiter des Departments Metallurgie der Montanuniversität Leoben

Bruno Hribernik

Geschäftsführer der ASMET

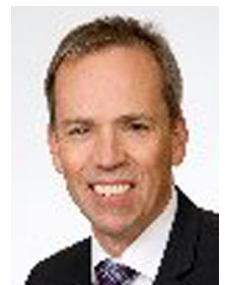

Johannes Schenk

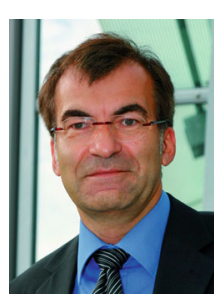

Bruno Hribernik 